\title{
Shear stress
}

To calculate the shear stress acting on the cells in the microfluidic channel, we solve the incompressible Navier-Stokes equation in 3D. The channel has a rectangular geometry $(x \in[0, L], y \in[-c, c], z \in[-b, b])$ and we use zero velocity boundary conditions at the boundaries of the channel cross section $(u(y= \pm c)=0$ and $u(z= \pm b)=0)$,

$$
\rho \frac{D u}{D t}=\rho g-\nabla p+\mu \nabla^{2} u .
$$

Here $\mu$ denotes fluid viscosity, $\rho$ fluid density, $p$ pressure, and $u$ fluid velocity. We solve using variable separation and Fourier series, and find an expression for the fluid velocity $u$

$$
\begin{aligned}
u & =\frac{G\left(b^{2}-z^{2}\right)}{2 \mu}+\sum_{n \text { odd }} A_{n} \cosh \left(\frac{n \pi}{2 b} y\right) \cos \left(\frac{n \pi}{2 b} z\right), \quad \text { with } \\
A_{n} & =-\frac{16 G b^{2}}{\mu \pi^{3} n^{3}} \frac{\sin \left(\frac{n \pi}{2}\right)}{\cosh \left(\frac{n \pi c}{2 b}\right)} .
\end{aligned}
$$

Where $G$ denotes the pressure drop in the channel. For a detailed derivation of the velocity please see [1].

We now calculate the wall shear stress from the velocity profile, using the expression

$$
\tau=\left.\mu \partial_{z} u\right|_{z=-c},
$$

and the velocity space derivative

$$
\partial_{z} u=-\frac{G z}{\mu}-\frac{n \pi}{2 b} \sum_{n \text { odd }} A_{n} \cosh \left(\frac{n \pi}{2 b} y\right) \sin \left(\frac{n \pi}{2 b} z\right) .
$$

Considering we do not have access to the pressure drop $G$ across the channel, but only the mean flow $Q$ we find an expression for the mean flow by integrating over the channel cross section,

$$
Q=\frac{G b^{3} c}{\mu}\left[\frac{4}{3}-\frac{8 b}{c} \sum_{n \text { odd }}\left(\frac{2}{n \pi}\right)^{5} \tanh \left(\frac{n \pi c}{2 b}\right)\right],
$$

thus we obtain the pressure drop $G$

$$
G=\frac{Q \mu}{b^{3} c}\left[\frac{4}{3}-\frac{8 b}{c} \sum_{n \text { odd }}\left(\frac{2}{n \pi}\right)^{5} \tanh \left(\frac{n \pi c}{2 b}\right)\right]^{-1} .
$$

We can simplify $G$ by using the values for $c$ and $b$. Note also that since $\pi c /(2 b) \approx 31.4$ the hyperbolic tangent becomes $\tanh (\pi c /(2 b)) \approx 1$, this leads to

$$
\begin{aligned}
G & =\frac{Q \mu}{b^{3} c} \times\left[\frac{4}{3}-\epsilon\right]^{-1} \quad \text { with } \\
\epsilon & =\frac{8 b}{c} \sum_{n \text { odd }}\left(\frac{2}{n \pi}\right)^{5} \tanh \left(\frac{n \pi c}{2 b}\right) \approx 4.2 \times 10^{-2} .
\end{aligned}
$$




\begin{tabular}{l|c}
$V_{f}\left[\frac{\mathrm{mm}}{\min }\right]$ & $\tau[\mathrm{Pa}]$ \\
\hline 1 & 0.000918 \\
5 & 0.004590 \\
10 & 0.009180 \\
15 & 0.013771 \\
20 & 0.018361 \\
30 & 0.027541 \\
50 & 0.045902
\end{tabular}

Table 1. Shear stress $\tau$ calculated as a function of imposed flow velocity $V_{f}$, using $\mu=8.9 \cdot 10^{-4} \mathrm{~Pa} \mathrm{~s}$, the dynamic viscosity of water and the channel dimensions $c=1$ $\mathrm{mm}$ and $b=0.05 \mathrm{~mm}$.

Then we can approximate this for small $\epsilon$

$$
G \approx \frac{Q \mu}{b^{3} c}\left[\frac{3}{4}+\left(\frac{3}{4}\right)^{2} \epsilon\right]
$$

Since we want to find the maximum stress, we choose $y$ to be at the middle of the channel $(y=0)$. Thus, we can simplify $\tau$ as well, $\operatorname{since} \cosh (n \pi y / 2 b)=1$, and $A_{n} \approx 0$ $\forall n \in \mathbb{N}$,

$$
\tau_{y=0}=-G z=-\frac{Q \mu z}{b^{3} c} \times\left[\frac{3}{4}+\left(\frac{3}{4}\right)^{2} \epsilon\right]
$$

We can now calculate the shear stress for the imposed mean flow velocities used in our experiments. An overview is shown in Table 1 .

Thus, the shear stresses are well below the critical value for the onset of mechanosensivity $0.7 \mathrm{~Pa}$ or even detachment 2.6 Pa 2 .

\section{References}

1. Vidal-Henriquez E, Zykov V, Bodenschatz E, Gholami A. Convective instability and boundary driven oscillations in a reaction-diffusion-advection model. Chaos: An Interdisciplinary Journal of Nonlinear Science. 2017;27(10):103110. doi:10.1063/1.4986153.

2. Décavé E, Rieu D, Dalous J, Fache S, Bréchet Y, Fourcade B, et al. Shear flow-induced motility of Dictyostelium discoideum cells on solid substrate. Journal of cell science. 2003;116(21):4331-4343. doi:10.1242/jcs.00726. 